\title{
Application of emerging technologies to improve access to ischemic stroke care
}

\author{
Shawn M. Vuong, MD,1,2 Christopher P. Carroll, MD,, Ryan D. Tackla, MD,1,2 \\ William J. Jeong, MD, ${ }^{1,2}$ and Andrew J. Ringer, MD'1-3 \\ ${ }^{1}$ Department of Neurosurgery, University of Cincinnati College of Medicine; ${ }^{2}$ Comprehensive Stroke Center at University of \\ Cincinnati Gardner Neuroscience Institute; and ${ }^{3}$ Mayfield Clinic, Cincinnati, Ohio \\ During the past 20 years, the traditional supportive treatment for stroke has been radically transformed by advances in \\ catheter technologies and a cohort of prominent randomized controlled trials that unequivocally demonstrated significant \\ improvement in stroke outcomes with timely endovascular intervention. However, substantial limitations to treatment \\ remain, among the most important being timely access to care. Nonetheless, stroke care has continued its evolution by \\ incorporating technological advances from various fields that can further reduce patients' morbidity and mortality. In this \\ paper the authors discuss the importance of emerging technologies-mobile stroke treatment units, telemedicine, and \\ robotically assisted angiography_as future tools for expanding access to the diagnosis and treatment of acute ischemic \\ stroke.
}

https://thejns.org/doi/abs/10.3171/2017.1.FOCUS16520

KEY WORDS acute ischemic stroke; mobile stroke treatment unit; robotic surgical procedure; telemedicine; health services accessibility

$\mathrm{U}$ NTIL the 1995 National Institute of Neurological Disorders and Stroke (NINDS) trial, ischemic stroke treatment consisted primarily of supportive care. ${ }^{30}$ After this landmark NINDS trial, intravenous (IV) tissue plasminogen activator (tPA) for ischemic stroke rapidly became the standard of care, thus ushering in a new era of stroke therapy. However, given the small therapeutic window, need for systemic administration, and poor recanalization of large arteries, IV-tPA faced numerous therapeutic limitations. The validation of intraarterial (IA) tPA provided an attractive treatment alternative because of higher rates of recanalization, lower effective dosages, and significantly lower rates of intracerebral hemorrhage. . $^{2,17,23,26,29,33}$

Unfortunately, IA-tPA did not significantly improve recanalization of large vessels. In an attempt to further improve large vessel recanalization, extend the therapeutic window, and facilitate treatment of patients with contraindications to thrombolysis, endovascular systems for mechanical and aspiration thrombectomy were developed. ${ }^{11,18,37}$ Early endovascular trials (i.e., Emergency Management of Stroke [EMS], Interventional Manage- ment of Stroke-I [IMS-I], and IMS-II) established the safety and feasibility of IA-tPA combined with mechanical thrombectomy. ${ }^{21,22,26}$ First-generation endovascular mechanical clot-retrieval systems did not show a significant improvement in cerebral revascularization rates or patient outcomes in the Mechanical Embolus Removal in Cerebral Ischemia (MERCI) and Multi-MERCI trials. ${ }^{18,44}$ Compared with the Second Prolyse in Acute Cerebral Thromboembolism Trial (PROACT II), trials of the Penumbra endovascular aspiration device similarly failed to demonstrate significant improvement in patient outcome scores, although significantly improved revascularization rates did result. ${ }^{35,45}$

With the improved technology of third-generation clot retrieval systems and greater facility with their use, 3 large randomized clinical trials were undertaken to validate endovascular thrombectomy in AIS. These landmark trials-Extending the Time for Thrombolysis in Emergency Neurological Deficits-IntraArterial (Extend IA), Endovascular Treatment for Small Core and Anterior Circulation Proximal Occlusion with Emphasis on Minimizing CT to Recanalization Times (ESCAPE), and Multi-

ABBREVIATIONS AIS = acute ischemic stroke; IA = intraarterial; IV = intravenous; MSTU = mobile stroke treatment unit; NINDS = National Institute of Neurological Disorders and Stroke; PHANTOM-S = Prehospital Acute Neurological Treatment and Optimization of Medical care in Stroke; RACA = robotically assisted cerebral angiography; RAll = robotically assisted intracranial intervention; tPA = tissue plasminogen activator. 
center Randomized Clinical Trial of Endovascular Treatment for Acute Ischemic Stroke in the Netherlands (MR CLEAN)-were closed prematurely when each achieved significant improvement in acute ischemic stroke (AIS) outcomes and vessel recanalization among those patients who underwent prompt endovascular intervention..$^{5,8,19}$ These trials finally and reproducibly established endovascular thrombectomy as a standard-of-care technique in the treatment of AIS.

The AIS treatment protocols from these studies were designed and implemented at comprehensive stroke centers or tertiary referral centers. Nationwide implementation of this new treatment paradigm faces numerous challenges. Arguably the greatest limiting factor is the time elapsed from symptom onset to pharmacological (IV-tPA) or endovascular treatment. Few patients $(3 \%-5 \%)$ present to medical care within the 4.5-hour treatment window for IV-tPA. ${ }^{2}$ Of those presenting for care from 2004 to 2009, fewer than $1 \%$ of patients with AIS received IA therapy. ${ }^{20}$ A second factor limiting widespread implementation is patient access to IV-tPA capable centers, let alone advanced endovascular treatment. In a study of the US population, Adeoye et al. reported that $19 \%$ reside more than 60 minutes from IV-tPA capable centers, half more than 60 minutes from endovascular-capable centers by ground transport, and 15\% more than 60 minutes from endovascular-capable centers by air transport (assuming its availability). ${ }^{1}$

In just over 2 decades, advancement in catheter-based endovascular technologies has revolutionized AIS care. These catheter technologies inspired a completely new paradigm for the treatment of AIS. However, access and expedient transport to advanced AIS care remain a major challenge to improving AIS outcomes for all patients. Helping to bridge this access-to-treatment gap are the development of telestroke programs, implementation of mobile stroke treatment units (MSTUs), and adoption of robotically assisted angiography technology.

\section{Mobile Stroke Treatment Units}

Each minute after AIS is associated with losses of an estimated 1.9 million neurons, 14 billion synapses, and 7.5 miles of myelinated fibers. Every 30-minute delay in revascularization leads to a $20 \%$ reduction in the likelihood of functional outcomes. ${ }^{31,41}$ These sobering statistics bring a cruel veracity to the cliché "time is brain." Today, the $3.4 \%-5.2 \%$ of patients with AIS in the US who receive IV-tPA during the 6-hour therapeutic window remains far below the optimal target of 30\%-50\% treatment rates, a statistic that demands improvement. ${ }^{2,36}$

A major factor in this low rate of IV-tPA for AIS is delayed presentation: many patients simply do not reach medical attention or are not assessed within the therapeutic window, $, 7,27$ Delayed presentation to medical care is a multifactorial issue. Importantly, many patients do not appreciate the seriousness of their symptoms or fail to recognize that they are having a stroke. Public health initiatives, such as the NINDS "Know Stroke" campaign, do help educate the public about the signs and symptoms of AIS. Appropriately trained health care providers at all levels of the treatment chain are essential to optimizing
AIS care. Diagnosis and screening can be a significant obstacle in reducing treatment times. Therefore, provider education is essential to facilitate the early, accurate diagnosis of AIS and the assessment of patient eligibility for tPA. The rise of prehospital MSTUs are leveraging technological innovations, such as portable CT scanners and high-speed wireless data transfer, to reduce patients' time to treatment. ${ }^{36}$

The MSTU, or the European Stroke Emergency Mobile (STEMO), is a means of reducing the delay in presentation of patients with AIS to trained AIS providers and could reduce the number of patients ineligible for tPA because of delayed presentation. An MSTU is a specialized ambulance equipped with a CT scanner, point-of-care laboratory capability, telemedicine connectivity, and medical staff specifically trained to care for AIS patients, including the administration of IV-tPA. ${ }^{36,46}$ This specialized, mobile stroke-response team brings AIS treatment and life-saving tPA to the patient rather than the patient needing to be transported to stroke providers. Ideally, this results in a shorter time to diagnosis, screening, and when applicable, IV-tPA administration. By these means, MSTUs may play a pivotal role in reducing the ischemic insult to the patient's brain, overall AIS morbidity and mortality, and societal cost for medical care, rehabilitation, and disability related to AIS. 3.16

After the idea for an MSTU was first proposed in Germany in 2003, the first MSTU became operational in Berlin in 2008. Data collected from early German MSTUs supported the feasibility of this mobile AIS treatment system and suggested MSTU deployment resulted in significantly decreased times to IV-tPA administration for the patient. ${ }^{16}$ In the US, the first MSTU was established at the University of Texas at Houston Medical Center in 2014 in collaboration with Frazer Ltd. and Neurologica, makers of the portable CT machine CereTom. ${ }^{24}$

A rapid succession of new MSTUs around the US followed: the Cleveland Clinic (2014), University of Colorado (Denver, 2015), Mercy St. Vincent Medical Center (Toledo, August 2015), University of Tennessee Health and Science Center (Memphis, 2016), and later in 2016 at both Rush University Medical Center (Chicago) and NewYorkPresbyterian Hospital (New York). These new MSTUs featured prominently in local, regional, and national news media and proved just as effective as their European counterparts. The Prehospital Acute Neurological Treatment and Optimization of Medical care in Stroke Study (PHANTOM-S) in Germany reported their MSTU significantly decreased stroke alert-to-treatment times from 76 minutes to 52 minutes, and significantly increased thrombolysis rates from $21 \%$ to $33 \%$ with no increase in the risk of intracerebral hemorrhage. ${ }^{15}$ Among patients receiving thrombolytic therapy, the same German group also reported the proportion of so-called "golden-hour thrombolysis" was 6-fold higher with MSTU deployment. ${ }^{14}$ In a re-analysis of PHANTOM-S data the Berlin group was unable to identify a significant improvement in hospital discharge disposition home $(\mathrm{p}=0.096)$ or in-hospital mortality ( $\mathrm{p}$ $=0.08)$ when MSTUs were deployed, citing only a trend toward improved outcomes. However, significantly fewer patients with AIS were transported to a facility without a Stroke Unit, and significantly fewer patients with intrace- 
rebral hemorrhage were transported to a facility without neurosurgical coverage $(\mathrm{p}<0.01){ }^{47}$ The Cleveland Clinic reported that in comparison with treatment before development of their MSTU, their patients received IV-tPA 40 minutes earlier with this unit, higher numbers of stroke alerts received IV-tPA, and time-to-imaging was reduced by 21 minutes. ${ }^{36}$

As the MSTU model continues to evolve, its further optimization will improve prehospital stroke treatment. For example, the University of Texas (Houston) is prospectively collecting long-term AIS and intracerebral hemorrhage patient outcome data to refine their use of an MSTU and optimize treatments. ${ }^{34}$ Furthermore, the advent of CT angiography-capable MSTUs in the US may ultimately streamline stroke care of patients with large vessel occlusion by bypassing hospitals and even stroke centers that lack endovascular capability. ${ }^{36}$ However, the capital investment required to implement and staff a full-time MSTU may prove prohibitive for many centers. ${ }^{34}$ Demonstration of improved patient outcomes and cost-effectiveness versus traditional prehospital stroke care are a prerequisite to widespread adoption of the MSTU model. Continued data from initial experiences with MSTUs and randomized trials of MSTU deployment versus traditional prehospital stroke care will help delineate the role of MSTUs and shape the landscape for prehospital stroke treatment in the coming years.

\section{Telemedicine}

Improvements in access to stroke treatment associated with MSTUs may be limited to large metropolitan areas where substantial resources for stroke care are readily available. This is problematic because much of the US population is geographically remote from certified stroke centers and "tPA ready" medical centers. Approximately $20 \%$ of the US population lives more than 60 minutes from hospitals capable of delivering IV-tPA. Even among hospitals with stroke treatment capabilities, treatment rates are low: only $4 \%$ of patients with AIS receive IV-tPA and $0.5 \%$ receive endovascular therapy. ${ }^{1}$ By leveraging highquality videoconferencing for face-to-face interactions and remote visualization of head CT scans, telestroke centers have shown promise in improving access to diagnosis and treatment of patients with AIS in rural areas. ${ }^{25,42}$

Using data from the US Census Bureau and geographic data system analyses, Samson et al. ${ }^{40}$ predicted that telestroke centers could significantly improve access to stroke care for the rural South Carolina population. For this specific population, $54 \%$ live within a 30 -minute drive and $77 \%$ live within a 60 -minute drive to a primary stroke center. By incorporating telestroke centers, South Carolina residents would have access to significantly more stroke care centers: $76 \%$ within 30 minutes and $95 \%$ within 60 minutes. Incorporating telestroke centers captures nearly a quarter more of the South Carolina population into the 30and 60-minute windows for diagnostic stroke evaluation.

The number of telestroke programs is steadily growing and providing community benefits, especially in rural areas with small hospitals. Key internal factors that drive the adoption of a telestroke program include enlarged stroke catchment area, improvements in clinical outcomes, in- creased clinical process efficiency, and community benefits. Telestroke programs have primarily been deployed for emergency room consultation, patient triage, and inpatient teleconsultations. Although these programs can improve access to AIS care, barriers to hospital adoption of telestroke programs and program sustainability remain. Among sites surveyed, frequently cited barriers to the maintenance or adoption of telestroke programs included the following: licensing/credentialing challenges; lack of technology support at remote sites; capital constraints; and trouble with physician acceptance or participation.,43

\section{Robotics}

Robotically assisted surgical devices have been used in operative theaters since 1983 when the Arthrobot, the world's first surgical robot, was introduced to assist with orthopedic procedures in Vancouver, Canada. In 1985, the Programmable Universal Machine for Assembly (PUMA) 560 system was used by neurosurgeons to robotically guide placement of brain biopsy needles. Subsequent types of surgical robots introduced in the 1990s were primarily experimental. However, this changed with the development of the first da Vinci surgical robotic system (Intuitive Surgical) in 2000, the first system approved by the FDA for general laparoscopic surgery. ${ }^{48}$ Due in large part to the da Vinci robotic system and adoption of robotically assisted surgery programs, significant strides were noted in urological, gynecological, and head and neck surgery in the $2000 \mathrm{~s}^{48}$

Around this time, vascular surgeons began investigating robotically assisted angiography to assist with the cannulation of aortic vessels. Engineering experience with the da Vinci medical robot spurred interest in the application of robotically assisted technologies for endovascular interventions. In 2002, Hansen Medical began working on an endovascular robotically assisted catheter system and received FDA approval in 2007 for the Sensei robotic system in ablative cardiac procedures. ${ }^{6}$ Early investigationsprimarily case reports-suggested that flexible robotically guided catheters can assist angiographers in the treatment of difficult pathologies. Animal studies were undertaken to compare the safety and feasibility of robotically assisted angiography with manual techniques. ${ }^{7,13}$ Beyond safety and feasibility, these studies suggested that robotically assisted catheter navigation could lessen endothelial trauma, which was hypothesized to potentially reduce the risk of arterial dissections and thromboembolic complications. ${ }^{7}$ Although robotic catheter navigation admittedly introduced more complexity, reports suggested a short learning curve for those well versed in manual catheter techniques. ${ }^{39}$

With the advent of navigable robotic catheters there has been evidence that these technologies allow endovascular interventionists to achieve stable sheath positions impossible with conventional manual techniques. ${ }^{9}$ As robotic-assistance technologies simplify complex procedures, some hypothesize that procedure times and total contrast dosages will decrease, which could then reduce anesthetic-, radiation-, and contrast-induced nephropathy risk. The prospect of using a protected robotic workstation distant from the radiation source may also benefit angiographers by reducing their radiation exposure. Furthermore, a ro- 


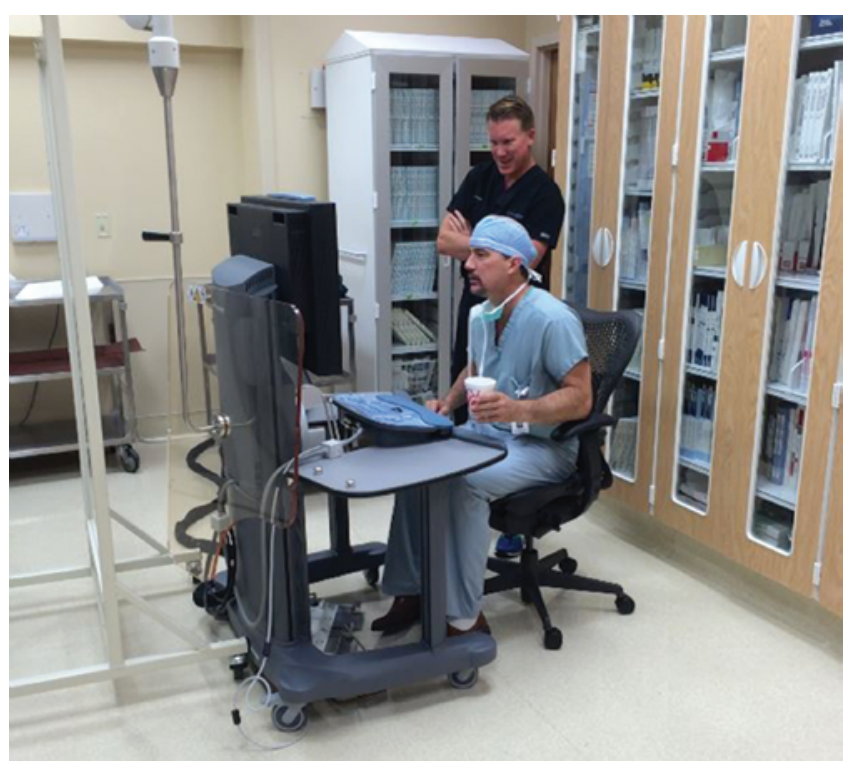

FIG. 1. Dr. Ringer performing a robotically assisted elective cerebral angiogram from the remote working station.

botically assisted catheter system may limit the fatigue associated with longer interventions by allowing the operator to be seated for significant portions of the procedure and obviating the need for protective lead clothing while at the remote workstation (Fig. 1). ${ }^{38}$ Perhaps the most exciting potential benefit of robotic angiography and intervention is the potential for remote operation by a stroke interventionist across a network of hospitals equipped with the nec- essary angiography infrastructure. This may dramatically broaden the geographic reach of each stroke interventionist, allowing patients with large vessel occlusions to reach a suitable treatment location even when the interventionist is farther away. Thus, the prospect of reducing transport times to reach a trained stroke interventionist could substantially impact clinical outcomes.

Robotically assisted angiography has been applied to both diagnostic and interventional management of extracranial vascular disease, but until recently it had not been described in the neurointerventional literature. ${ }^{3,6,28,38,39} \mathrm{Lu}$ et al. reported their initial experience with robotically assisted diagnostic angiography in 15 patients using the Vascular Interventional Robot (VIR-2; Navy General Hospital of People's Liberation Army, Beijing University, China). The authors reported a mean $( \pm$ SD) robotically assisted diagnostic angiography time of $34.4 \pm 5.13$ minutes and reported no periprocedural complications. Furthermore, as robotic assistance allowed for remote operation, staff radiation exposure was limited to the time needed for femoral artery access. ${ }^{28}$

We began using the Magellan Robotic Catheter System (Hansen Medical) to perform endovascular procedures in 2015. Our robotically assisted procedures begin with manual femoral artery access and placement of a 9-Fr short femoral sheath. The robotic catheter is then placed into the femoral artery over an extra-long guidewire (Fig. 2); at this point all further manipulations-including those of the guidewire - can be performed from the remote robotic workstation (Fig. 3). The robotic catheter we use for cerebral angiography consists of a flexible outer guide catheter (9-Fr outer diameter, 6-Fr inner diameter, up to $90^{\circ}$ of

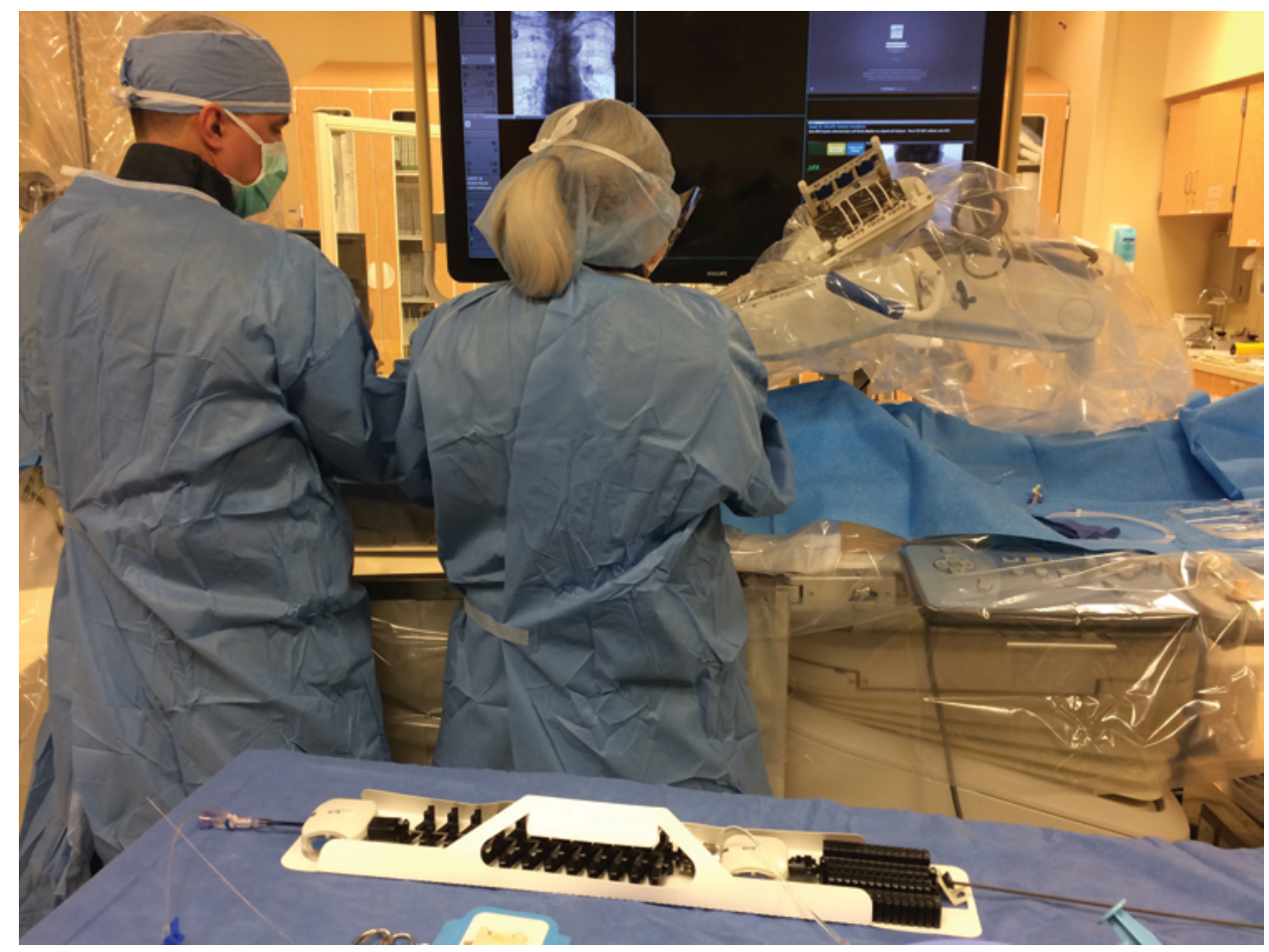

FIG. 2. Dr. Carroll setting up the Magellan Robotic System after femoral access for RACA. The robotic arm is visible in the right upper quadrant; the robotically assisted guide catheter is noted in the foreground. 


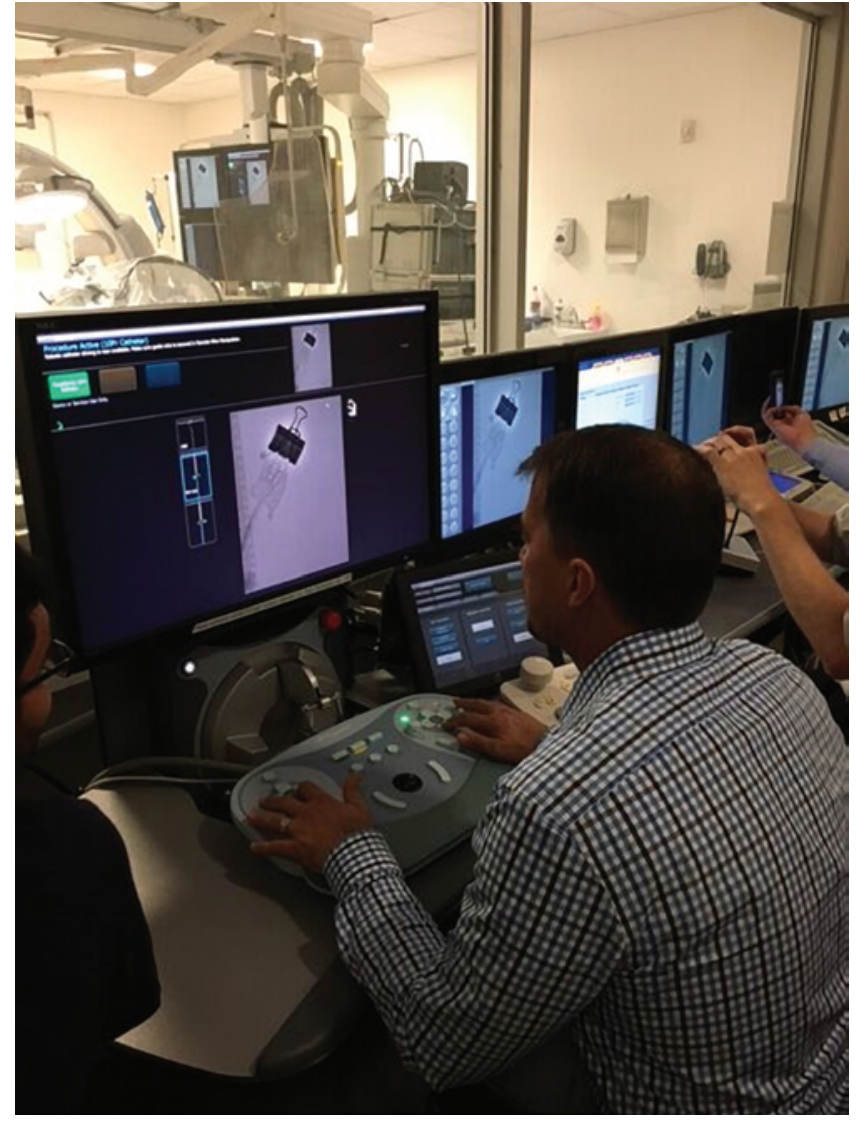

FIG. 3. Dr. Ringer at the control panel of the Magellan remote robotic workstation.

articulation) and flexible inner leader catheter (6-Fr outer diameter, $0.03^{\prime \prime}$ inner diameter, up to $180^{\circ}$ of articulation). During robotically assisted cerebral angiography (RACA), the guide catheter is typically parked in the aortic arch and articulated along the inner curvature of the aorta, directed toward the target vessel; the leader catheter is then advanced to cannulate the target vessel and anchored proximally for diagnostic angiography (Fig. 4). For robotically assisted intracranial intervention (RAII) the procedure is identical except the guide catheter is navigated along the leader catheter to cannulate the target vessel and the leader catheter is withdrawn once the guide is optimally positioned. Once the leader catheter is withdrawn, up to 2 microcatheters can then be deployed via the guide catheter. At completion of RACA or RAII, the femoral artery access site is closed with an 8-Fr Angio-Seal closure device (St. Jude Medical).

We prospectively evaluated our initial institutional experience with RACA and RAII using the Magellan Robotic catheter system. During a 15-month period (20152016), 23 consecutive patients underwent a total of 9 RACA and 18 RAII procedures. These robotically assisted procedures were compared with 7 manual diagnostic or interventional procedures performed in the same patient cohort. Compared with matched angiography controls, we found no significant differences in procedure time, fluoroscopy times, or contrast volumes for RACA ( $p>0.05$ ). After the senior author's (A.J.R.) first 5 procedures, differ-

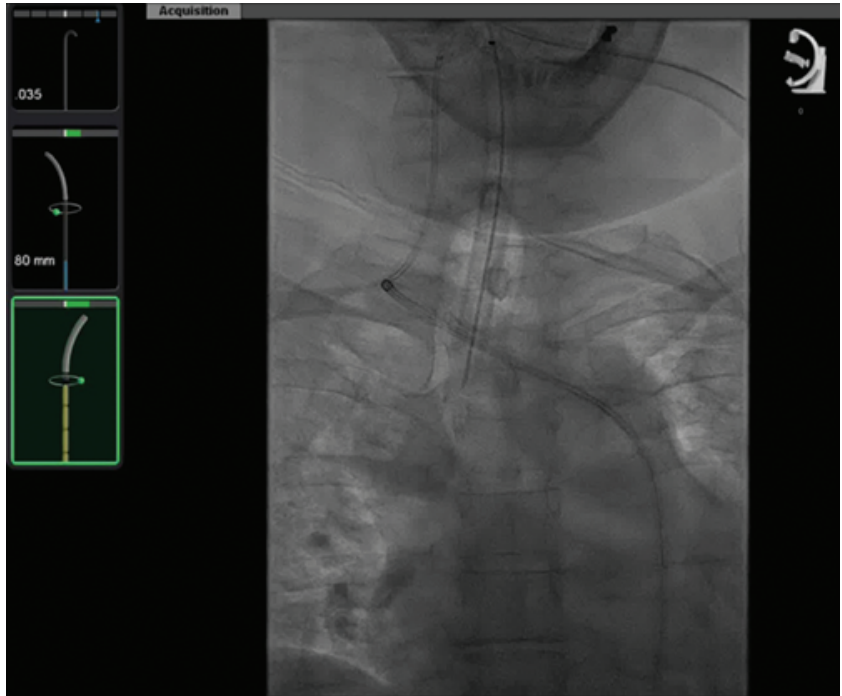

FIG. 4. Fluoroscopic and catheter display of the Magellan remote robotic workstation during diagnostic cerebral angiography. The guide catheter is observed within the aortic arch; the leader catheter can be seen cannulating the right common carotid artery.

ences between manual and robotically assisted procedure and fluoroscopy times became negligible; no perioperative complications occurred. We concluded that use of the Magellan robotically assisted catheter system for cerebral angiography and cerebrovascular interventions was technically feasible without an apparent increased risk to the patient. After a short learning curve, procedure time, fluoroscopy time, and contrast volumes did not differ significantly from manually performed diagnostic cerebral angiography controls (Carroll CP et al., unpublished data, 2016). ${ }^{10}$

Robotically assisted endovascular interventions are still in their infancy, particularly cerebral angiography and interventions. Following what we believe to be the first and second safety and feasibility case series in RAII and RACA, respectively, ${ }^{28}$ ongoing collection of safety data will be essential to clarify the role for robotically assisted catheter systems in the endovascular suite of the future. In peripheral artery disease, the Robotic Vascular and Endovascular Registry was designed to prospectively evaluate the safety, feasibility, and efficacy of this system. With further prospective data to validate safety and efficacy, a randomized trial comparing robotically assisted and manual endovascular angiography and interventions is the next logical step.

Interventions for AIS could benefit greatly from robotically assisted technology. As noted above, these technologies can not only improve the speed of complex procedures but can potentially reduce endothelial damage, contrast doses, and radiation exposure. With further technological refinement, additions might include fiberoptic endovascular angioscopy with the possibility for direct visualization of thrombectomy with mechanical, aspiration, ultrasonic, or a combination of these techniques. ${ }^{32}$ With the stability of a robotically assisted catheter system, the potential for remote operation of the system could greatly expand access to stroke interventionalists, allowing patients to receive thrombectomy from a distant location. 


\section{Conclusions}

The treatment paradigm for AIS has evolved dramatically during the past 2 decades. With improved endovascular techniques and evidence from recent publications of 3 landmark trials, endovascular thrombectomy is an integral part of AIS patient care. However, these treatment paradigms were designed for comprehensive stroke centers and face many challenges to broad adoption nationwide. Patient access and expedient transport for AIS diagnosis and treatment remains one of the great challenges for the large portions of the US population that live more than 60 minutes from the nearest stroke center. Emerging technologies, such as MSTUs, telemedicine, and RACA may significantly decrease access impediments for rural patients. Growing evidence of the critical nature of "door-to-needle" times has promoted the development of MSTUs with early evidence supporting improved stroke treatment provision where MSTUs are deployed. MSTUs may prove pivotal in optimizing prehospital stroke care across the nation, particularly in areas remote from a comprehensive stroke center. Similarly, telemedicine has shown significant promise for improving access to AIS diagnosis and treatment for rural patients by leveraging high-quality videoconferencing and teleradiology technologies. The advent of robotically assisted endovascular technologies, although in their infancy, also shows promise in decreasing complex procedure times and reducing endothelial damage. Furthermore, robotically assisted endovascular technologies may provide the platform for further technical advances for AIS care, such as angioscopy, endovascular microsurgery, and remote operation to improve revascularization rates and access to a stroke interventionalist. With continued refinement and elaboration of these promising technologies, the future of acute stroke care looks bright.

\section{Acknowledgments}

We would like to thank Mary Kemper, medical editor for the University of Cincinnati/Mayfield Clinic Department of Neurosurgery, for her assistance in the formatting and editing of this manuscript.

\section{References}

1. Adeoye O, Albright KC, Carr BG, Wolff C, Mullen MT, Abruzzo T, et al: Geographic access to acute stroke care in the United States. Stroke 45:3019-3024, 2014

2. Adeoye O, Hornung R, Khatri P, Kleindorfer D: Recombinant tissue-type plasminogen activator use for ischemic stroke in the United States: a doubling of treatment rates over the course of 5 years. Stroke 42:1952-1955, 2011

3. Antoniou GA, Riga CV, Mayer EK, Cheshire NJW, Bicknell CD: Clinical applications of robotic technology in vascular and endovascular surgery. J Vasc Surg 53:493-499, 2011

4. Barber PA, Zhang J, Demchuk AM, Hill MD, Buchan AM: Why are stroke patients excluded from TPA therapy? An analysis of patient eligibility. Neurology 56:1015-1020, 2001

5. Berkhemer OA, Fransen PSS, Beumer D, van den Berg LA, Lingsma HF, Yoo AJ, et al: A randomized trial of intraarterial treatment for acute ischemic stroke. N Engl J Med 372:11-20, 2015

6. Bismuth J, Duran C, Stankovic M, Gersak B, Lumsden AB: A first-in-man study of the role of flexible robotics in overcoming navigation challenges in the iliofemoral arteries. J Vasc Surg 57 (2 Suppl):14S-19S, 2013
7. Bismuth J, Kashef E, Cheshire N, Lumsden AB: Feasibility and safety of remote endovascular catheter navigation in a porcine model. J Endovasc Ther 18:243-249, 2011

8. Campbell BCV, Mitchell PJ, Kleinig TJ, Dewey HM, Churilov L, Yassi N, et al: Endovascular therapy for ischemic stroke with perfusion-imaging selection. N Engl J Med 372:1009-1018, 2015

9. Carrell T, Dastur N, Salter R, Taylor P: Use of a remotely steerable "robotic" catheter in a branched endovascular aortic graft. J Vasc Surg 55:223-225, 2012

10. Carroll C, Tackla R, Vuong S, Jeong W, Serrone J, Ringer A: Robotic-assisted cerebral angiography and intracranial intervention: initial experience with the Magellan Catheter System. J Neurosurg 126:A1431, 2017 (Abstract)

11. Christou I, Alexandrov AV, Burgin WS, Wojner AW, Felberg RA, Malkoff M, et al: Timing of recanalization after tissue plasminogen activator therapy determined by transcranial Doppler correlates with clinical recovery from ischemic stroke. Stroke 31:1812-1816, 2000

12. del Zoppo GJ, Higashida RT, Furlan AJ, Pessin MS, Rowley HA, Gent M: PROACT: a phase II randomized trial of recombinant pro-urokinase by direct arterial delivery in acute middle cerebral artery stroke. PROACT Investigators. Prolyse in Acute Cerebral Thromboembolism. Stroke 29:4-11, 1998

13. Duran C, Lumsden AB, Bismuth J: A randomized, controlled animal trial demonstrating the feasibility and safety of the Magellan $^{\mathrm{TM}}$ endovascular robotic system. Ann Vasc Surg 28:470-478, 2014

14. Ebinger M, Kunz A, Wendt M, Rozanski M, Winter B, Waldschmidt C, et al: Effects of golden hour thrombolysis: a Prehospital Acute Neurological Treatment and Optimization of Medical Care in Stroke (PHANTOM-S) substudy. JAMA Neurol 72:25-30, 2015

15. Ebinger M, Winter B, Wendt M, Weber JE, Waldschmidt C, Rozanski M, et al: Effect of the use of ambulance-based thrombolysis on time to thrombolysis in acute ischemic stroke: a randomized clinical trial. JAMA 311:1622-1631, 2014

16. Fassbender K, Walter S, Liu Y, Muehlhauser F, Ragoschke A, Kuehl S, et al: "Mobile stroke unit" for hyperacute stroke treatment. Stroke 34:e44, 2003

17. Furlan A, Higashida R, Wechsler L, Gent M, Rowley H, Kase $\mathrm{C}$, et al: Intra-arterial prourokinase for acute ischemic stroke. The PROACT II study: a randomized controlled trial. Prolyse in Acute Cerebral Thromboembolism. JAMA 282:20032011, 1999

18. Gobin YP, Starkman S, Duckwiler GR, Grobelny T, Kidwell CS, Jahan R, et al: MERCI 1: a phase 1 study of Mechanical Embolus Removal in Cerebral Ischemia. Stroke 35:28482854, 2004

19. Goyal M, Demchuk AM, Menon BK, Eesa M, Rempel JL, Thornton J, et al: Randomized assessment of rapid endovascular treatment of ischemic stroke. N Engl J Med 372:10191030,2015

20. Hassan AE, Chaudhry SA, Grigoryan M, Tekle WG, Qureshi AI: National trends in utilization and outcomes of endovascular treatment of acute ischemic stroke patients in the mechanical thrombectomy era. Stroke 43:3012-3017, 2012

21. IMS Study Investigators: Combined intravenous and intraarterial recanalization for acute ischemic stroke: the Interventional Management of Stroke Study. Stroke 35:904-911, 2004

22. IMS II Trial Investigators: The Interventional Management of Stroke (IMS) II Study. Stroke 38:2127-2135, 2007

23. Keris V, Rudnicka S, Vorona V, Enina G, Tilgale B, Fricbergs $\mathrm{J}$ : Combined intraarterial/intravenous thrombolysis for acute ischemic stroke. AJNR Am J Neuroradiol 22:352-358, 2001

24. Lake DM: UTHealth introduces nation's first Mobile Stroke 
Unit. UTHealth. February 3, 2014. (https://www.uth.edu/ media/story.htm?id=b1485cfc-110f-4a4c-91ea-06b573b3ba6d) [Accessed February 8, 2017]

25. Levine SR, Gorman M: "Telestroke": the application of telemedicine for stroke. Stroke 30:464-469, 1999

26. Lewandowski CA, Frankel M, Tomsick TA, Broderick J, Frey J, Clark W, et al: Combined intravenous and intra-arterial r-TPA versus intra-arterial therapy of acute ischemic stroke: Emergency Management of Stroke (EMS) Bridging Trial. Stroke 30:2598-2605, 1999

27. Lichtman JH, Watanabe E, Allen NB, Jones SB, Dostal J, Goldstein LB: Hospital arrival time and intravenous t-PA use in US Academic Medical Centers, 2001-2004. Stroke 40:3845-3850, 2009

28. Lu WS, Xu WY, Pan F, Liu D, Tian ZM, Zeng Y: Clinical application of a vascular interventional robot in cerebral angiography. Int J Med Robot 12:132-136, 2016

29. Macleod MR, Davis SM, Mitchell PJ, Gerraty RP, Fitt G, Hankey GJ, et al: Results of a multicentre, randomised controlled trial of intra-arterial urokinase in the treatment of acute posterior circulation ischaemic stroke. Cerebrovasc Dis 20:12-17, 2005

30. Marler JR, Welch KMA, Tilley BC, Brott T, Lyden P, Grotta JC: Tissue plasminogen activator for acute ischemic stroke. $\mathbf{N}$ Engl J Med 333:1581-1587, 1995

31. Mazighi M, Chaudhry SA, Ribo M, Khatri P, Skoloudik D, Mokin M, et al: Impact of onset-to-reperfusion time on stroke mortality: a collaborative pooled analysis. Circulation 127:1980-1985, 2013

32. McVeigh PZ, Sacho R, Weersink RA, Pereira VM, Kucharczyk W, Seibel EJ, et al: High-resolution angioscopic imaging during endovascular neurosurgery. Neurosurgery 75:171180,2014

33. Ogawa A, Mori E, Minematsu K, Taki W, Takahashi A, Nemoto S, et al: Randomized trial of intraarterial infusion of urokinase within 6 hours of middle cerebral artery stroke: the middle cerebral artery embolism local fibrinolytic intervention trial (MELT) Japan. Stroke 38:2633-2639, 2007

34. Parker SA, Bowry R, Wu TC, Noser EA, Jackson K, Richardson L, et al: Establishing the first mobile stroke unit in the United States. Stroke 46:1384-1391, 2015

35. Penumbra Pivotal Stroke Trial Investigators: The penumbra pivotal stroke trial: safety and effectiveness of a new generation of mechanical devices for clot removal in intracranial large vessel occlusive disease. Stroke 40:2761-2768, 2009

36. Rasmussen PA: Stroke management and the impact of mobile stroke treatment units. Cleve Clin J Med 82 (12 Suppl 2):S17-S21, 2015

37. Rha JH, Saver JL: The impact of recanalization on ischemic stroke outcome: a meta-analysis. Stroke 38:967-973, 2007

38. Riga CV, Bicknell CD, Rolls A, Cheshire NJ, Hamady MS: Robot-assisted fenestrated endovascular aneurysm repair (FEVAR) using the Magellan system. J Vasc Interv Radiol 24:191-196, 2013

39. Riga CV, Bicknell CD, Sidhu R, Cochennec F, Normahani $\mathrm{P}$, Chadha P, et al: Advanced catheter technology: is this the answer to overcoming the long learning curve in com- plex endovascular procedures. Eur J Vasc Endovasc Surg 42:531-538, 2011

40. Samson M, Trivedi T, Heidari K: Telestroke centers as an option for addressing geographical disparities in access to stroke care in South Carolina, 2013. Prev Chronic Dis 12:E227, 2015

41. Saver JL: Time is brain-quantified. Stroke 37:263-266, 2006

42. Schwamm LH, Holloway RG, Amarenco P, Audebert HJ, Bakas T, Chumbler NR, et al: A review of the evidence for the use of telemedicine within stroke systems of care: a scientific statement from the American Heart Association/ American Stroke Association. Stroke 40:2616-2634, 2009

43. Silva GS, Farrell S, Shandra E, Viswanathan A, Schwamm LH: The status of telestroke in the United States: a survey of currently active stroke telemedicine programs. Stroke 43:2078-2085, 2012

44. Smith WS, Sung G, Saver J, Budzik R, Duckwiler G, Liebeskind DS, et al: Mechanical thrombectomy for acute ischemic stroke: final results of the Multi MERCI trial. Stroke 39:1205-1212, 2008

45. Tarr R, Hsu D, Kulcsar Z, Bonvin C, Rufenacht D, Alfke K, et al: The POST trial: initial post-market experience of the Penumbra system: revascularization of large vessel occlusion in acute ischemic stroke in the United States and Europe. J Neurointerv 2:341-344, 2010 (Erratum in J Neurointerv 3:97, 2011)

46. Walter S, Kostopoulos P, Haass A, Keller I, Lesmeister M, Schlechtriemen T, et al: Diagnosis and treatment of patients with stroke in a mobile stroke unit versus in hospital: a randomised controlled trial. Lancet Neurol 11:397-404, 2012

47. Wendt M, Ebinger M, Kunz A, Rozanski M, Waldschmidt C, Weber JE, et al: Improved prehospital triage of patients with stroke in a specialized stroke ambulance: results of the pre-hospital acute neurological therapy and optimization of medical care in stroke study. Stroke 46:740-745, 2015

48. Yates DR, Vaessen C, Roupret M: From Leonardo to da Vinci: the history of robot-assisted surgery in urology. BJU Int 108:1708-1714, 2011

\section{Disclosures}

The authors report no conflict of interest concerning the materials or methods used in this study or the findings specified in this paper.

\section{Author Contributions}

Conception and design: Ringer. Acquisition of data: Ringer, Carroll. Analysis and interpretation of data: all authors. Drafting the article: all authors. Critically revising the article: all authors. Reviewed submitted version of manuscript: all authors. Statistical analysis: Ringer, Carroll. Study supervision: Ringer.

\section{Correspondence}

Andrew J. Ringer, Department of Neurosurgery, University of Cincinnati College of Medicine, 260 Stetson St., ML 670515, Cincinnati, OH 45267-0515. email: aringer@mayfieldclinic.com. 